\section{Prevalencia de dengue y leptospirosis en pacientes febriles en un hospital general de especialidades, México}

\author{
Suemy Cen, Selene Blum-Domínguez, Luis Núñez-Oreza, \\ Fredy Díaz, Betty Sarabia, Paulino Tamay-Segovia
}

Prevalence of dengue and leptospira in febrile patients of the general hospital of specialities in Mexico

Among the infectious diseases characterized by a febrile picture are: dengue, leptospirosis, rickettsiosis and salmonellosis, among others. The objective of this study was to identify IgM antibodies against dengue and Leptospira in febrile patientes. The seropositivity for IgM antibodies to dengue was $34 \% ; 26.3 \%$ for women and $7.6 \%$ for men, without differences significant $(\mathrm{p}=0.181)$. The seropositivity for IgM antibodies to Leptospira was 3.2\%, being found only in women. Positive serology for both dengue and Leptospira was $1 \%$. The serovars detected were Pomona and Canicola by the microagglutination technique. It was possible to identify the unsuspected presence of leptospirosis and dengue in atypical months for the latter, an important fact to consider the study of serology in the differential diagnosis of febrile diseases.

Key words: Leptospirosis; dengue; febrile patients.

Palabras clave: Leptospirosis; dengue; pacientes febriles.

\section{Introducción}

Las infecciones caracterizadas por cuadros febriles con sintomatología inespecífica son frecuentes en los trópicos ${ }^{1}$. Entre estas enfermedades febriles se encuentra el dengue, leptospirosis, rickettsiosis y salmonelosis, entre otras ${ }^{2,3}$. La fiebre es un motivo de consulta frecuente y que se encuentra presente en distintas patologías. En zonas endémicas es necesario realizar un inmunodiagnóstico para identificar el agente y brindar el tratamiento correcto. Un mal diagnóstico puede llevar a un manejo inadecuado y causar la muerte del paciente, principalmente en infecciones con escasa sospecha clínica como la rickettsiosis o leptospirosis. En México y otros países existe un subdiagnóstico de leptospirosis debido a su mimetismo clínico con dengue y otras enfermedades febriles ${ }^{4}$. Respecto a dengue, en los estados mexicanos que reportaron su presencia, la circulación es continua ${ }^{5}$. Campeche se localiza en el sureste de México, tiene una temperatura promedio anual de $26^{\circ} \mathrm{C}$ en todo el Estado y $28^{\circ} \mathrm{C}$ en la costa y con una precipitación promedio anual de $1.120 \mathrm{~mm}$.

Centro de Investigaciones Biomédicas, Universidad Autónoma de Campeche, Campeche, México (SC, SBD, LNO, PTS).

Hospital General de Especialidades “Dr. Javier Buenfíl Osorio" (FD).

Facultad de Medicina, Universidad Autónoma de Campeche, Campeche, México (BS).

Los autores declaramos no tener conflictos de interés.

El proyecto fue financiado por la Universidad Autónoma de Campeche.

Recibido: 22 de agosto de 2017 / Aceptado: 22 de marzo de 2018

Correspondencia a:

Paulino Tamay-Segovia

pautamay@hotmail.com
El objetivo de este estudio fue identificar anticuerpos IgM a dengue y Leptospira en pacientes febriles de la localidad de Campeche.

\section{Material y Métodos}

Se realizó un estudio observacional, transversal y descriptivo en el Hospital General de Especialidades de la ciudad de Campeche, entre febrero y julio de 2015. Se obtuvieron sueros de pacientes que acudieron a consulta con tres a cinco días de $\mathrm{T}^{\mathrm{o}}$ axilar mayor a $37,5^{\circ} \mathrm{C} \sin$ foco y como único tratamiento antiinflamatorios no esteroidales. Estos pacientes fueron remitidos entre los ocho y 12 días de fiebre al laboratorio de enfermedades febriles del hospital en que se utilizó un kit de antígenos febriles Licon, que incluye Salmonella spp., Proteus (OX-19) (el cual presenta reacción cruzada para bacterias del género Rickettsia) y Brucella spp. Los pacientes participaron previo consentimiento informado, posteriormente se les aplicó un cuestionario epidemiológico. Se excluyeron a los pacientes con síndrome febril con foco conocido. El suero se obtuvo de una muestra de sangre sin anticoagulante por venopunción. Una alícuota de cada suero se trasladó al laboratorio del Centro de Investigaciones Biomédicas. Se determinaron anticuerpos IgM para dengue y Leptospira empleando el kit IgM ELISA comercial de DRG (DRG Instruments GmbH, Germany) con una sensibilidad y especificidad de $100 \%$ y una sensibilidad de $100 \%$ y una especificidad del $89,7 \%$, respectivamente. De acuerdo a las instrucciones del fabricante se definieron las muestras positivas y negativas utilizando los controles del kit. El serovar causal de leptospirosis se determinó por la prueba de microaglutinación. Los datos fueron analizados usando frecuencias simples, proporciones, $\mathrm{y} \mathrm{chi}^{2}$ como medida de asociación entre seropositividad y género. Este estudio fue evaluado y aprobado por el Comité de Ética en Investigación de la Secretaría de Salud del Gobierno del estado de Campeche.

\section{Resultados}

Se estudiaron un total de 91 muestras de pacientes febriles de 3 a 87 años de edad, 65 mujeres y 26 varones. Treinta (32,9\%) resultaron positivas a uno o más antígenos en la prueba de reacciones febriles: $10,9 \%$ para Salmonella spp., 1\% a Brucella spp. y 20,8\% Proteus (OX-19).

Seropositividad a dengue: $34 \%$ (31/91) de las muestras fueron positivas para anticuerpos IgM a dengue. El 26,4\% en mujeres de 6 a 58 años y 7,6\% en varones de 18 a 61 años. Aunque se encontró una mayor seroprevalencia en mujeres, no hubo una diferencia significativa $(p=0,181)$. El rango de edad con mayor seropositividad fue de 51-61 años, tanto para mujeres como para hombres. El 64,5\% (20/31) de las muestras positivas se colectaron en los meses de sequía.

Seropositividad a leptospirosis: 3,2\% (3/91) fueron positivos para anticuerpos IgM a Leptospira spp., todas mujeres entre 33 y 52 años. Los serovares detectados fueron Pomona (33,3\%) y Canicola (66,6\%). El 1\% (1/91) presentó serología positiva a Leptospira y dengue; 63,7\% (58/91) fueron negativas a estas pruebas.

\section{Discusión}

En este estudio se detectaron anticuerpos IgM a Leptospira similar a lo encontrado por Rodríguez-Villamarín ${ }^{6}(2,9 \%)$ y ligeramente menor a la 
registrada por Navarrete-Espinosa ${ }^{7}(4 \%)$ y Becerra $^{8}(5,8 \%)$. Esta seropositividad predominó en mujeres al igual que en Itapúa, Paraguay ${ }^{9}$, y diferente a los resultados obtenidos por Céspedes ${ }^{10}$ que obtuvo una seropositividad similar entre hombres y mujeres. Los serovares detectados fueron Pomona y Canicola, lo cual concuerda con lo reportado por Sánchez-Barragán ${ }^{11}$ y Pulido-Medellín ${ }^{12}$. El serovar Canicola asociado a perros fue el que se encontró con mayor frecuencia, tal como en Yucatán ${ }^{13}$. Aunque con una frecuencia baja, la leptospirosis es evidente en la población febril estudiada. Esta se puede adquirir por el contacto con la orina infectada de roedores y animales domésticos, debido a esto las mujeres son las más propensas puesto que permanecen mayor tiempo en las viviendas.

Por otra parte, la frecuencia de anticuerpos IgM a virus dengue fue $34 \%$, mayor a la reportada en Itapúa (25\%), donde no se encontró diferencia entre varones y mujeres. En nuestro estudio hubo predominio en mujeres porque fueron las más estudiadas. Navarrete y cols. también reportó una seroprevalencia ligeramente mayor en mujeres que en hombres. En relación a la edad, observamos mayor reactividad en los mayores de 50 años, tanto en varones como en mujeres, en congruencia con Navarrete-Espinosa ${ }^{7}$. Esto puede deberse a los hábitos peri-domiciliarios del mosquito lo que ocasiona una mayor exposición de las mujeres, así como la mayor edad. El 1\% de las muestras resultaron positivas para dengue y leptospirosis, similar a lo encontrado en Venezuela ${ }^{14}$, Veracruz ${ }^{7}$ e Itapúa ${ }^{9}$. Llama la atención que la mayor proporción de muestras positivas se colectaron en los meses de sequía, lo que indica una circulación permanente del virus dengue ${ }^{5}$.

Este perfil etiológico revela que $69,2 \%$ de los pacientes presentó positividad para alguno de los agentes etiológicos estudiados. En el resto sin agente causal identificado, la enfermedad febril pudo deberse a otras infecciones como paludismo, rickettsiosis y bartonelosis ${ }^{15}$. Por otra parte, una limitante del estudio fue no poder obtener una segunda muestra para confirmar los resultados negativos y una rickettsiosis.

En conclusión, nuestro estudio permitió identificar la presencia insospechada de leptospirosis y dengue en temporada atípica para este último. Esto puede dificultar el diagnóstico correcto, debido al mimetismo clínico entre estas infecciones. Recomendamos que la detección de estos anticuerpos debe ser incluida en el diagnóstico diferencial de las enfermedades febriles en zonas endémicas.

Agradecimientos: A las Q.F.B. Adda Ortiz López y Raquel Castillo Rodríguez del Hospital General de Especialidades "Dr. Javier Buenfil Osorio" de la ciudad de Campeche, México.

\section{Resumen}

Entre las enfermedades infecciosas febriles se encuentran: dengue, leptospirosis, rickettsiosis y salmonelosis, entre otras. El objetivo de este estudio fue detectar la presencia de anticuerpos IgM a dengue y Leptospira en pacientes febriles. La seropositividad para IgM frente al dengue fue $34 \% ; 26,3 \%$ en mujeres y $7,6 \%$ en varones, sin diferencias significativas ( $\mathrm{p}=0,181)$. La seropositividad para los anticuerpos IgM a Leptospira fue 3,2\%; encontrándose sólo en mujeres. La serología positiva para leptospirosis y dengue fue $1 \%$. Los serovares detectados fueron Pomona y Canicola por la técnica de microaglutinación. Se pudo identificar la presencia insospechada de leptospirosis y dengue en meses atípicos para este último, hecho importante para considerar el estudio de serología en el diagnóstico diferencial de enfermedades febriles.

\section{Referencias bibliográficas}

1.- Victoriano A F, Smythe L D, Gloriani-Barzaga N, Cavinta L L, Kasai T, Limpakarnjanarat K, et al. Leptospirosis in the Asia Pacific region. BMC Infect Dis 2009; 9: 147. doi: 10.1186/1471-2334-9-147.

2.- Leelarasamee A, Chupaprawan C, Chenchittikul M, Udompanthurat S. Etiologies of acute undifferentiated febrile illness in Thailand. J Med Assoc Thai 2004; 87: 464-72.

3.- Pradutkanchana J, Pradutkanchana S, Kemapanmanus M, Wuthipum N, Silpopajakul K. The etiology of acute pyrexia of unknown origin in children after a flood. Southeast Asian J Trop Med Public Health 2003; 34: 175-78.

4.- Vanasco N B, Schmeling M F, Chiani Y, Lottersberger J, Tarabla H D. Diagnóstico de leptospirosis humana: evaluación de la aglutinación macroscópica en diferentes etapas de la enfermedad. Salud Publica Mex 2012; 54: 530-6.

5.- Torres-Galicia I, Cortés-Poza D, Becker I. Dengue en México: incremento en la población juvenil durante la última década. Bol Med Hosp Infant Mex 2014; 71: 196-201. doi: 10.1016/j.bmhimx.2014.08.003.

6.- Rodríguez-Villamarín F R, Prieto-Suárez E, Escandón P L, De la Hoz Restrepo F. Proporción de leptospirosis y factores relacionados en pacientes con diagnóstico presuntivo de dengue, 2010-2012. Rev Salud Publica (Bogota) 2014; 16: 597-609.

7.- Navarrete-Espinosa J, Acevedo-Vales J A, Huerta-Hernández E, TorresBarranca J, Gavaldón-Rosas D G. Prevalencia de anticuerpos contra dengue y Leptospira en la población de Jáltipan, Veracruz. Salud Publica Mex 2006; 48: $220-8$.

8.- de Melo Bezerra L F, Fontes R M, Gomes A M, da Silva D A, Colares J K, Lima D M. Serological evidence of leptospirosis in patients with a clinical suspicion of dengue in the State of Ceará, Brazil. Biomedica 2015; 35: 55762. doi: 10.7705/biomedica.v35i4.2504.

9.- Cabello M A, Cabral M B, Samudio M, Páez M, Jiménez R, Arce M, et al. Dengue y leptospirosis compartiendo el mismo nicho ecológico en la localidad ribereña de Carmen del Paraná (Itapúa). Mem Inst Investig Cienc Salud 2010; 8: 35-40.

10.- Céspedes M, Ormaeche M, Condori P, Balda L, Glenny M. Prevalencia de leptospirosis y factores de riesgo en personas con antecedentes de fiebre en la Provincia de Manu, Madre de Dios, Perú. Rev Peru Med Exp Salud Publica 2003; 20: 180-5.

11.- Sánchez-Barragán B, Velasco-Castrejón O, Rivas-Sánchez B, FernándezRíos M P, Alvarado-Vigil P E, Márquez-Cruz M. Leptospirosis en trabajadores de campos petroleros de Tabasco. Salud en Tabasco 2017; 23: 19-27. https://tabasco.gob.mx/sites/default/files/users/ssaludtabasco/19.pdf

12.- Pulido-Medellín M, Díaz-Anaya A, Giraldo-Forero J. Determinación de Leptospira spp. en humanos y bovinos pertenecientes al municipio de Toca, Boyacá. Veterinaria y Zootecnia 2017; 11: 55-66. doi: 10.17151/ vetzo.2017.11.2.5

13.- Zavala-Velázquez J E, Vado-Solís I A, Rodríguez-Félix M E, RodríguezAngulo E M. Barrera-Pérez M A, Guzmán-Marín E S. Leptospirosis anictérica en un brote epidémico de dengue en la Península de Yucatán. Rev Biomed 1998; 2: 78-83.

14.- López E, Moros R, Cardona M, Zambrano J, Morón D, Hernández R, et al. Hallazgo de infecciones concomitantes leptospira-fiebre amarilla y leptospira-dengue en casos de pacientes fallecidos con síndrome febril icterohemorrágico en Venezuela años 2000-2004. Bol Venez Infectol 2006; 17: 8-12.

15.- Troyes L, Fuentes L, Troyes M, Canelo L, García M, Anaya E, et al. Etiología del síndrome febril agudo en la providencia de Jaén, Perú 20042005. Rev Peru Med Exp Salud Publica 2006; 23: 5-11. 